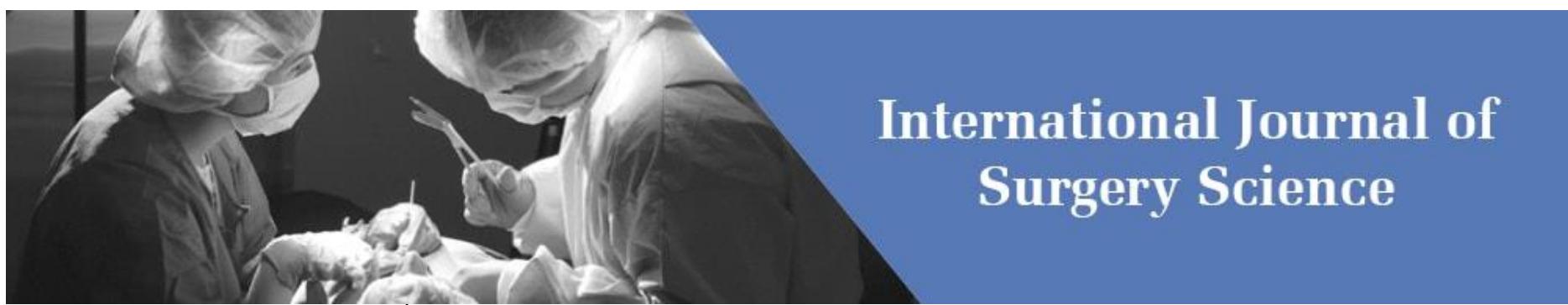

E-ISSN: 2616-3470

P-ISSN: 2616-3462

(C) Surgery Science

www.surgeryscience.com

2020; 4(1): 244-246

Received: 01-11-2019

Accepted: 05-12-2019

Dr. Lamia Inayath

Post Graduate, Department of

General Surgery, Yenepoya

Medical College and Hospital,

Deralakatte, Mangalore,

Karnataka, India

Dr. Krishnakanth

Senior Resident, Department of General Surgery, Yenepoya

Medical College and Hospital,

Deralakatte, Mangalore,

Karnataka, India
Corresponding Author: Dr. Krishnakanth

Senior Resident, Department of

General Surgery, Yenepoya

Medical College and Hospital,

Deralakatte, Mangalore,

Karnataka, India

\section{Correlation between clinical diagnosis and colonoscopic findings of patients presenting with lower gastrointestinal bleeding}

\author{
Dr. Lamia Inayath and Dr. Krishnakanth
}

DOI: https://doi.org/10.33545/surgery.2020.v4.i1e.340

\section{Abstract}

AIMS and Objectives: The purpose of the study was to explore the causes of lower GI bleeding and correlate them with colonoscopic findings.

Materials and Methods: In this study, a total of 80 patients who presented to Yenepoya medical college from January 2019 to September 2019 with at least one episode of per rectal bleeding were taken and colonoscopy was performed for all patients. The clinical diagnosis at the time of presentation and the colonoscopic findings were compared and the results tabulated.

Results: The most common causes of lower GI bleeding included haemorrhoids, colitis and colorectal malignancies. Colonoscopy findings were found to be normal only in $2.8 \%$ of patients, as compared to $22.8 \%$ on clinical diagnosis. The sensitivity and specificity of the clinical symptoms and signs was very low. The clinical diagnosis made was correct in $40 \%$ of the cases.

Conclusion: 1) The more frequent endoscopic findings were colitis, colorectal malignancies and polyps. 2) Clinical data are of little value in predicting a normal examination. 3) Total colonoscopy appears to be the first procedure of choice in all patients with lower gastrointestinal bleeding, irrespective of the clinical data and the presence of anal pathology. 4) Symptoms are unhelpful in deciding who requires investigation.

Keywords: lower Gi bleeding, colonoscopy

\section{Introduction}

Lower GI bleeding (LGIB) is historically defined as bleeding that emanates from a source distal fto the ligament of Treitz. After the advent of deep enteroscopy, small bowel sources have been placed under the category of mid gut bleeding and a new definition of LGIB has been proposed as bleeding distal to the ileocaecal valve ${ }^{[1,2,4]}$. Acute LGIB is defined as bleeding of recent duration ( $<3$ days) that may lead to haemodynamic instablilty, anemia or the need for blood transfusions. Chronic LGIB is defined as passage of blood per rectum over several days or longer, and usually implies slow or intermittent loss of blood ${ }^{[1]}$. The majority of patients with lower gastrointestinal (G.I.) hemorrhage will stop bleeding during resuscitation. Investigation for the source of the bleed can then proceed with endoscopic and radiographic studies, followed by definitive procedure, if indicated.

\section{Materials and Methods}

This observational study included patients presenting to Yenepoya Medical college and Hospital from January 2019 to September 2019 with at least 1 episode of bleeding per rectum. Per rectal examination and proctoscopy was performed and a clinical diagnosis was made prior to the procedure.

\section{Inclusion criteria}

- Male and female patients with age above 18 yrs.

- Patients presenting with visible bleeding per rectum as their main complaint

Exclusion criteria

- Patients below the age of 18 yrs.

- Patients with possible upper gastrointestinal bleeding site, i.e., history of 
haematemesis/malena or vomiting/naso-gastric aspirates containing coffee-ground material or fresh blood.

- Patients with suspected peritonitis.

- Patients with any history of coagulopathy.

- Those patients who did not consent or refused colonoscopy.

Informed written consent was taken from each patient before the interview according to the guidelines of the hospital. All the information of the patients was kept confidential. Concerned authorities were also informed and consent was obtained. Medical management consisted of hospitalization, monitoring, and resuscitation in intensive care. Anticoagulants and nonsteroidal anti-inflammatory drugs, including aspirin, were discontinued before colonoscopy. Patients also received transfusions of red cells/packed cells for severe anaemia before they underwent urgent colonoscopy. All the patients were prepared for Colonoscopy by asking them to use liquid only daily for three days prior to the examination. They were administered enema twice on the night before and early morning in the day of the procedure. Colonoscopic results were compiled and analysed using the statistical tests. Lesions with some degree of suspicion were biopsied and sent to laboratory for histo-pahological studies.

\section{Results}

Table 1: Gender characteristics

\begin{tabular}{|c|c|c|}
\hline & Number & Percent \\
\hline Female & 30 & $37.5 \%$ \\
\hline Male & 50 & $62.5 \%$ \\
\hline Total & 80 & 100 \\
\hline
\end{tabular}

Of the patients who presented with LGIB, $62.5 \%$ were males and $37.5 \%$ were females.

Table 2: PR Findings

\begin{tabular}{|c|c|c|}
\hline & Number & Percent \\
\hline Haemorrhoids & 41 & 51.25 \\
\hline Fissures & 7 & 8.75 \\
\hline Growth & 11 & 13.75 \\
\hline Polyp & 5 & 6.25 \\
\hline Colitis & 3 & 3.75 \\
\hline Normal & 13 & 16.25 \\
\hline Total & 44 & 100 \\
\hline
\end{tabular}

Based on clinical findings and per rectal examination and proctoscopy, $51.25 \%$ of the patients included in the study were found to have haemorrhoids.

Only 3 patients was found to have colitis on per rectal examination and proctoscopy.

Table 3: Comparison of Colonoscopic and clinical findings

\begin{tabular}{|c|c|c|}
\hline & $\begin{array}{c}\text { Clinical } \\
\text { diagnosis }\end{array}$ & $\begin{array}{c}\text { Colonoscopic findings } \\
\text { (additional findings) }\end{array}$ \\
\hline Haemorrhoids & $41(51.25 \%)$ & $23(28.75 \%)$ \\
\hline Fissures & $7(8.75 \%)$ & $5(6.25 \%)$ \\
\hline Growth & $11(13.75 \%)$ & $21(26.25 \%)$ \\
\hline Normal & $13(16.25 \%)$ & $2(2.5 \%)$ \\
\hline Colitis & $3(3.75 \%)$ & $23(28.75 \%)$ \\
\hline Polyps & $5(6.25 \%)$ & $11(13.75 \%)$ \\
\hline
\end{tabular}

Following colonoscopy, the common causes of LGIB were haemorrhoids, malignant growth, colitis, polyps, and fissures. On comparison of clinical diagnosis with colonoscopic findings, it was found that of the 41 patients found to have haemorrhoids on clinical findings, only 23 patients were confirmed to have only haemorrhoids. 18 patients were found to have a second pathology, such as colitis, malignant growth and polyps. On clinical diagnosis, 13 patients were found to have normal per rectal and proctoscopic examination, wheras on colonoscopy, 11 patients were found to have a second pathology in the colon. This proves that clinical diagnosis hold little value in the final diagnosis of lower GI bleeding. Symptoms and signs are least decisive in determining who requires investigation.

\section{Colonosopy findings of various causes of lower gi bleeding}

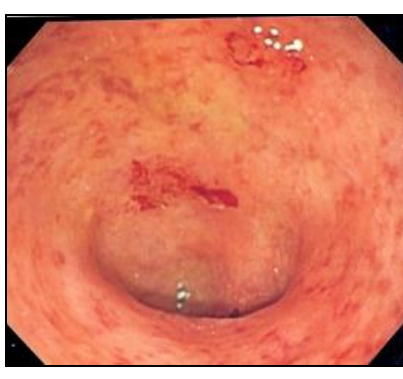

Colitis

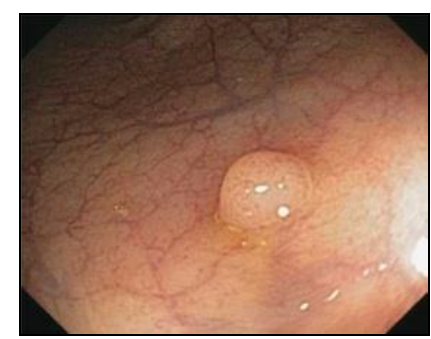

Polyp

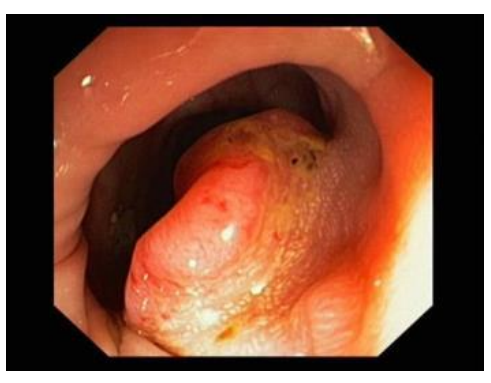

Ulceroproliferative Growth

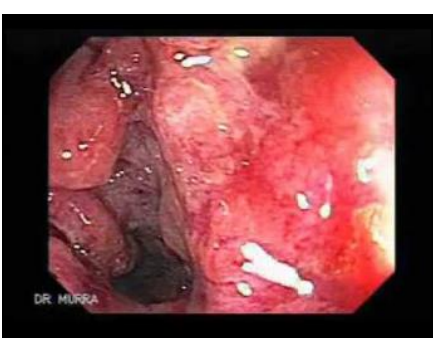

Haemorrhoids

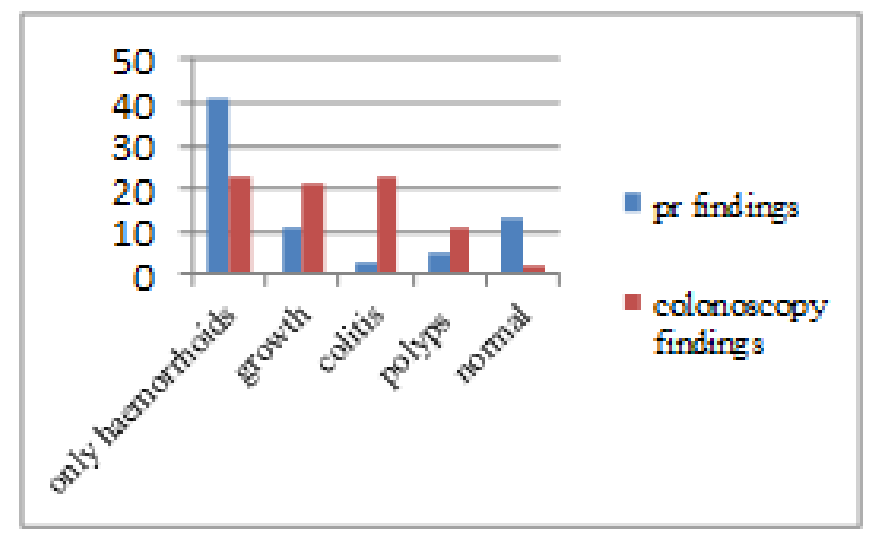

\section{Discussion}

One of the frequent causes of hospital admission is LGIB and it is a major factor that is involved in hospital morbidity \& mortality. LGIB is different from upper GI bleeding in epidemiology, prognosis, and management ${ }^{[1,4,5]}$. Symptoms of haemorrhoids are also associated with other abnormalities ${ }^{[2,3]}$. It is feasible to exclude these before concluding that haemorrhoids are responsible for the presenting complaints. It is stated that a full colonoscopy is necessary in all patients, regardless the age ${ }^{[1,}$ ${ }^{5,6]}$. In common practice, the age of 50 years holds the threshold for colonoscopy since the incidence of colorectal cancer, diverticulosis and polyps increases as people are getting older ${ }^{[1,}$ $2,3,4]$. It is important to inspect the rectum and colon because significant findings proximal to the anal canal can be present in patients with haemorrhoids especially in older patients ${ }^{[4]}$. 
It is not possible, merely from a per rectal examination, to be certain that rectal bleeding necessarily originates from a simple rectal lesion, such as haemorrhoids ${ }^{[3,4,5]}$. These lesions may coexist with colorectal adenoma or cancer ${ }^{[2,5,7]}$.

\section{Conclusion}

In this study, haemorrhoids and colitis were the leading causes for lower GI bleeding. Other causes included colorectal malignancies and polyps.

Coincidental findings in the colon and rectum of patients with haemorrhoids are often present, especially in older patients. These may have important consequences, hence patients presenting with LGIB should undergo full examination of the colon.

\section{References}

1. Causes of lower gastrointestinal bleeding on colonoscopy, $\mathrm{J}$ Ayub Med Coll Abbottabad Rehman Jehangiri, 2017; 29(3).

2. Incidence and causes of rectal bleeding in general practice as detected by colonoscopy, British Journal of General Practice, Metcalf. 1996; 46:161-164,

3. Research article A survey of abnormalities in the colon and rectum in patients with haemorrhoids, Koning and Loffeld BMC Gastroenterology Mark V Koning and Ruud JLF Loffeld. 2010; 10:74.

4. Diagnostic work-up of rectal bleeding in general practice, British Journal of General Practice, January Heintze, 2005.

5. Correlation between Clinical Diagnosis and Colonoscopic Findings of Patients Presented with Lower Gastrointestinal Bleeding, Mymensingh Med J Rahman MM. 2015; 24(2):238-43.

6. Acute and chronic management of lower gastrointestinal bleeding: cost-effective approaches, The Gastroenterologist [01 Sep], Machicado GA, Jensen DM. 1997; 5(3):189-201.

7. Diagnosis of gastrointestinal bleeding: A practical guide for clinicians, World J Gastrointest Pathophysiol. Bong. 2014; $15,5(4): 467-478$. 